\title{
A Review on Capacitive Liquid Level Sensing Techniques
}

\author{
Nikhil Chandra B S ${ }^{1 *}$, Roopa $\mathrm{J}^{2}$, Harigovind $\mathrm{A}^{3}$ and Ajay Bharadwaj ${ }^{4}$ \\ ${ }^{1,2} R$ V College of Engineering, Bengaluru, India \\ ${ }^{3,4}$ Cypress Semiconductor, An Infineon Technologies Company, Bengaluru, India \\ ${ }^{1}$ nikhilchandrabs.ec17@rvce.edu.in, ${ }^{2}$ roopaj@rvce.edu.in, ${ }^{3}$ A.Harigovind@infineon.com, \\ ${ }^{4}$ Ajay.Bharadwaj@infineon.com
}

\begin{abstract}
For contemporary communities, liquid level management is of great significance. Liquid-level monitoring is utilised in a variety of industrial applications, including food processing, pharmaceutical manufacturing, chemical manufacturing, and water purification systems. Liquids are used in critical applications such as rocket fuel tanks, medical equipment, etc. These systems are prone to accidents caused by liquid leakages and liquid turbulence. Hence it is necessary to prevent such mishaps and save resources and additional costs which are incurred due to the same. This necessitates design and development of liquid level sensing systems which are used to detect and monitor the level of liquid. There are many techniques which can be used to sense the level of liquid like mechanical floats, ultrasonic sensors, fibre optic cables, LASER systems, light dependant resistors, image processing, etc. Capacitive sensing has emerged as one of the booming technologies due to its simplistic design, responsiveness, accuracy, noise immunity and easy deployment. It has evolved over time and is now a vital feature of a variety of products. This paper aims to review the existing capacitive sensing mechanisms and attempts to serve as a foundation for unexplored sensor geometries. A comparative analysis has been presented to assess the performance of the proposed techniques. Coplanar capacitive sensors are found to be effective over cylindrical capacitive sensors due to decrease in size, simple design and cost effectiveness while maintaining the same accuracy, resolution and sensitivity.
\end{abstract}

Keywords: Capacitive sensing, Coplanar geometry, Cylindrical geometry, Interdigitated capacitive sensor, Liquid level measurement.

\section{Introduction}

Liquid level sensing is an indispensable and a requisite part of many industrial applications. Liquid level sensing is used in industries such as the automobile sector, home appliances, health care, milk and beverage, etc. It is mainly employed in liquid level detection and liquid leakage detection applications. The liquid level detection system is ideally required to be minimalistic, easy to deploy, accurate, power efficient and economic. Liquid level sensing can be implemented using various technologies such as ultrasonic sensing, millimetre-wave sensing, mechanical components, optic fibres etc.

A mechanical system would involve the usage of floats, motors, and additional equipment to automate the system. This would make the system bulky and expensive. Ultrasonic [1] and millimetre wave [2] techniques require frequency precision sources to generate the waves. Ultrasonic sensing technique also requires equipment to accurately measure the time of flight i.e., the time taken for the wave to travel to-and-fro the source/sensor. This time duration will be very small, in the order of micro to nanosecond, and thus the measurement is error-prone. Doppler effect and temperature drifts make the readings further deviate from the desired output. The mechanical structure of the container and the presence of 
gas bubbles in the liquid contribute towards wave scattering. Even though energy is sufficient for the waves to suffer multiple reflections and reach the sensor (receiver), the time of flight calculated would be incorrect, leading to wrong prediction of liquid level.

Ultrasonic sensor fails when sensing is required through an opaque material. Millimeter waves [2] penetrate through opaque substances, however, exhibit a wide beam spread angle. A vibrator is required to amplify and reflect a portion of the millimeter waves received. The millimeter-wave source too involves many blocks/stages to generate the waves, thus making the system bulky and expensive. SomchatSonasang et al. in [3] demonstrated the usage of microstrip parallel coupled lines that come in contact with the liquid to detect its level. The frequency response of coupling was performed at frequencies ranging from $\mathrm{MHz}$ to GHz. The above-mentioned techniques/researches require sources to generate frequency precise signals which are proven and evident to be power guzzlers.

In this paper, capacitive sensing technique, which overcomes the above-mentioned pitfalls, has been centre staged. Extensive study about the evolution and the current state-ofthe-art techniques for liquid level sensing are presented.

\section{Historical implementation of liquid level sensors}

Liquid level sensing has been implemented for various applications ranging from interplanetary to household applications. For many years, liquid level sensing has been used. A notable method to detect the level of liquid was designed and developed by Greenwood et al. in the year 1958 [4]. Discrete and continuous liquid oxygen level detection has been proposed [4]. An insulated wire of thin diameter was left to float on the liquid surface with the help of two supports. When the liquid level changes, the vertical position of the wire changes. This contributes for change in resistance of the circuit. Wilner et al. in 1960 developed a capacitive measurement system to monitor red fuming nitric acid and slosh level in liquid propellant tanks of a rocket [5]. The sensing element, a Kel-F [5] insulated wire, was fixed along the walls of the acid tank. Capacitance would be formed when this insulated wire is dipped in the reactive acid. Toth et al. had proposed a novel method of capacitive sensing involving a segmented sensor array guarded on either sides by guard sensors [6]. Microwave techniques to monitor the liquid surface level were proposed by Stuchly et al. in [7]. The proposal uses bulky blocks such as vector voltmeter to measure wave phase and input reflection coefficient measurement system. Longrigg et al. in [8] have developed a liquid level sensor using laser technology. Helium-Neon laser was passed through the container holding the liquid, and upon encountering the fluid menisci, the laser would be deflected from its straight path.

Various methods, similar to the above mentioned researches, had been explored in this regard. It can be noticed that these methods involve voluminous and power consuming components. The requirement of additional precision measurement instruments increases the cost too. Capacitive sensing has emerged as a prominent technology in the recent years for its simplistic design, responsiveness and high sensitivity.

\section{Review}

\subsection{Capacitive sensors - cylindrical geometry}

Capacitive sensing systems provide a superlative solution to detect the level of the liquid. Bera et al. have focussed on developing cylindrical capacitive sensors [9]. Extensive research has been done by them. In [9], a cylindrical capacitive sensor has been developed, 
which gets attached as an extension to the container. The sensing cylinder is made up of insulating material, with two sets of windings around it. The core of the cylinder is hollow into which liquid can enter. It is assumed that the liquid is grounded as the container used is metallic. The liquid column forms one electrode and the copper windings form the other electrode. The insulating cylinder present in between these two electrodes forms the dielectric material. The sensing coil is protected from interference from electromagnetic radiation by a grounded coaxial metal screen. The capacitance readings were measured using the modified De'Sauty bridge, consisting of a Wein bridge oscillator and two operational amplifiers. The same circuit was proposed for grounded metallic container too. The experiment was conducted using both metallic and non-metallic containers holding conducting liquid. The presence of grounded coaxial metal screen makes the parasitic capacitance of the system constant. Measurements of liquid level were reported with errors of $4 \%$ and $1 \%$ for metallic and non-metallic containers respectively.

Bera et al. in [9] failed to account for variation of parasitic capacitance due to atmospheric properties, close proximity of other conductors near the sensing system, etc. Chakraborty et al. in [10] have overcome this by considering four identical non-inductive coil capacitors. This technique employs measuring differential capacitances. This is measured with two different setups. The first setup involves measuring differential capacitance between tube filled with liquid and tube filled with air. The second setup involves measuring differential capacitance between tube filled with liquid and tube filled with both liquid and air. These two differential capacitances, when considered together, have proven to be independent of parasitic capacitance variation. The output was observed to vary linearly with the level of liquid in that container. But the proposed system requires mechanical alterations and support system. The sensor system cannot be used as a plug-and-play device.

Jin et al. in [11] have proposed a system based on grounded cylindrical capacitive sensor, much similar to the sensor proposed by Bera et al. in [9]. But Jin et al. have developed a backwards difference calculation (of the sensor readings) without any extra overhead to reduce the effects of changes in parasitic capacitance. A novel technique employing active shield was used such that it has no effect on the parasitic capacitance. The inner cylinder of the active shielded cable is connected to ground and the exterior cylinder is utilized for liquid level measurement. Good performance with error percentage of only $0.675 \%$ was reported for a measuring range of $200 \mathrm{~cm}$.

The conventional capacitive sensing for liquid level detection consists of a metallic rod dipped inside the liquid. Metallic rod acts as an electrode and the walls of the vessel act as the other electrode. This is the case when the vessel is conductive or metallic. If the vessel is non-conductive, two metallic rods are used to serve as the two electrodes of the capacitor. The capacitance measured from either of the systems is directly proportional to the amount of liquid present in the vessel, or in other words, the level of the liquid, as the dielectric constant of the liquid influences the amount of capacitance of the system. AC bridge circuit system is generally used to measure the capacitance in these conventional capacitive sensing systems. Magnetic field exists around the metallic rod when subjected to alternating current. This magnetic field defines the self-inductance of the metallic rod, and this is nonlinearly dependent on current density, frequency of alternating current, etc. Bera et al. [12] have proposed a capacitive type liquid level sensor which eliminates the self-inductance property of the metallic rod.

The sensing system consists of two concentric cylinders made up of insulating material. Each cylinder is wound with two layers of non-inductive windings, thus eliminating any development of self-inductance. The concentric cylinders are separated by another cylinder placed in between them. Air space is present between the inner cylinder and the intermediate cylinder. The setup is held rigid with a strong but perforated Teflon bottom 
plate. Hence, when this system is dipped inside the liquid, the latter can enter the air space present in the former, enabling 360-degree interaction of the cylinders with the liquid. The cylinders formed out of windings may be considered as continuous solid cylinders if thick windings having thin insulation are used. This ensures that the resolution of the abovedescribed sensor is in range/comparable with the conventional capacitive sensor. Two different readings were recorded, namely capacitance readings from an LCR meter and voltage readings from a De-Sauty bridge for incremental liquid levels. The sensor output was proven to linearly vary with the level of liquid, with very less deviation from the linear response. The proposed sensor may be used for conductive or non-conductive liquids present in conductive or nonconductive containers. But the proposed system is bulky to be installed in space critical applications.

SiddharthBardiya et al. in [13] have analysed the design proposed by Bera et al. [12] under different orientations of the container. The container, along with the sensor system, was kept on an inclined wedge, whose angle could be varied incrementally. The experiment was performed under normal conditions, with positive slope (inclination) and with negative slope (declination). Impedance analyzer was used to capture the capacitance readings and the errors (in percentage) for different angles of inclination were recorded. Considerable deviation from the expected liquid level was observed when the inclination was not flat (normal), with error percentages reaching as high as $20 \%$.

Guirong et al. in [14] developed a capacitive sensing system consisting of four electrodes. The proposed sensor design is unique as it can be modified into two electrode mode and four electrode mode. The four electrodes are stuck on the inner walls of a cylinder. The system can detect not only the level of liquid present in the container but the gradient of the container itself. Further, the angle and direction of the gradient of the container can be determined. In the twoelectrode system, adjacent sensors C1 and C2 are connected, and C3 and C4 are connected. The capacitance between the sensors "electrodes" is directly proportional to the level of liquid. In the four-electrode system, capacitance values are read between adjacent sensors. Four capacitance readings are obtained and in order to determine the container gradient, differences between adjacent capacitance values are calculated. These differences are considered as components of a $2 \mathrm{D}$ vector. The magnitude of the vector is the gradient angle and the slope of the vector is the gradient direction. The sensor system was tested for seven discrete liquid levels. Four different gradient angles and gradient directions each were chosen to evaluate the performance of the system.

For the two-electrode system, the capacitance values did not exhibit considerable variation beyond the gradient angle of $30^{\circ}$. The gradient direction of the container did not contribute to changes in capacitance; hence the two-electrode system is suitable to determine liquid level while being unrelated to the gradient of the vessel. The four-electrode system is capable to determining the gradient angle and gradient direction in real-time. It was proven that the gradient measurements are independent of the liquid level. The performance was not good for high gradient angles beyond $45^{\circ}$.

Usually, the capacitive sensors are exposed to air if they aren't interacting with liquids or interact with the level of air that isn't occupied by the liquid. The dielectric constant of air largely varies with temperature and humidity levels. Liquids exhibiting large dielectric constant than air do not face or face negligible effect due to this varying dielectric constant of air. This becomes a parameter to be considered for liquids with lower dielectric constants. HuseyinCanbolat in [15] has proposed a three-sensor system to eliminate the effects of varying dielectric constant of air. It is also independent of the properties of the liquid and a mathematical model has been proposed to support the claims. A reference sensor is placed at the bottom of the container, so that it can always interact with the liquid. An air sensor, with the same spatial dimensions, is symmetrically placed at the top of the container. This is 
intended to interact only with air. A level sensor, which runs through the height of the container, reports the level of liquid present in the container. Capacitive to digital converter blocks are used to get digital outputs from the sensed capacitance.

Good performance was reported for non-conducting liquids using the three-sensor system. The error was reduced to the order of $10^{-14} \mathrm{~m}$. The performance depends on the width and thickness of the sensors. The reference sensor cannot serve its purpose unless it is completely submerged inside the liquid, thus constraining the system to work with a minimum required liquid level above zero reading. The reference sensor dimensions could change due to liquid pressure as it always needs to be exposed to the liquid. This could be overcome by having thick sensors, but there arises a possibility for the electric field lines in leaving the container dimensions, thus exposing the electric field to two different media. This defeats the purpose of the sensor being constantly exposed to only the liquid. This is an area in which research is still being carried out.

Additionally, the sensor is required not to react with the liquid. An extra exterior protective coating is necessary to avoid the same. The air sensor is used to account for the varying dielectric constant of air. Certain liquids may vaporize and be present in the air just above the liquid surface. Different levels of air may be exposed to different concentrations of the vaporized liquid. However, the simulation was carried out assuming uniform distribution of vaporized liquid in the air present above liquid surface. Lastly, few modifications are proposed to the three-sensor system in [15] to suit level detection for conducting liquids. This requires the sensing system to be present in a flexible container, always filled with nonconducting liquid. This introduces additional mechanical requirements and makes the system cumbersome.

\subsection{Capacitive sensors - coplanar geometry}

Coplanar sensors feature the various parts of the sensor in a single plane. Capacitive coplanar sensor is very different from the conventional parallel plate capacitor. The former is usually manufactured on the outer surface of a printed circuit board (PCB). This layer gets exposed to the medium in which the sensing needs to be performed. Comb design realised in coplanar structure is one of the widely used capacitive sensors [16]. It consists of two sets of electrodes, one for transmitting and the other for receiving signals. The electrodes of these two sets are arranged in alternating fashion and the mutual capacitance is evaluated between the electrodes or "fingers" of the comb drive. Electric field lines exist between the electrodes. These field lines pass through the environment to which the coplanar sensor is exposed to. Thus, the properties of the environment affect the measured capacitance.

The dielectric constant of the environment surrounding the sensor plays a major role in determining the measured capacitance. Capacitance varies directly with respect to dielectric constant. Dielectric constant may vary along with the properties of the medium. If the medium is air, then the humidity level causes variation in dielectric constant of air [16]. Temperature and salinity modifies the dielectric constant of a liquid. Thus, it is important to consider the properties of the liquid and the medium in which the sensor system is exposed to.

In liquid level measurement, the amount of capacitance depends upon the extent till which the electric field lines penetrate into the liquid. This is constrained by the material and thickness of the container. The amount of capacitance measured also depends upon the area of each electrode. Pietrikova et al. [16] implemented three different coplanar capacitive comb sensors with width of electrode being 175 micrometer and electrode spacing being 200 micrometer. These sensors, which differ from each other in the effective sensor area, were tested for their performance using distilled water and transformer oil. It was experimentally proven that sensitivity (change in capacitance per unit length) is directly proportional to the 
amount of overlap of the electrodes present in the coplanar comb sensor structure. The sensitivity is, however, constrained by the limiting factor of the electrode overlap area. Upon reaching the limiting factor, any further increase in the electrode overlap area does not significantly contribute for increase in sensitivity, and the same can be eliminated from the design.

Capacitive liquid level sensing can also be used to monitor the quality of liquids. The resistivity of the liquid affects the measured capacitance. Resistivity of the liquid depends on the presence of ions and impurities. Since the measured capacitance is the change in capacitance and not the absolute capacitance, it is possible to use capacitive coplanar comb sensors in liquid contamination sensing too.

Many researchers have developed interdigitated capacitive sensors (or coplanar capacitive sensors) which work on the principle of fringing field. Li et al. in [17] developed a new fringing field capacitive sensor. Fringing field is a result of non-ideal obeyance of boundary conditions. When two conductors of a capacitor under the influence of applied potential difference are placed close to each other, the charges reside on the outer surface and closer to the other conductor. If a parallel plate capacitor is considered, then all the charges have to ideally be present on the flat surface faced to the other electrode. Hence the field lines should originate only from the above said flat surface. Since edges have larger curvature than the flat surfaces, the amount of charge accumulated at these edges are high. Field lines can be observed to fringe away from the edges of the conductor electrode. When the area of the capacitor plate is doubled, the field lines originating from the flat surface also gets doubled, but the fringing field remains constant. Hence fringing field is non-linearly related to the total capacitance of a sensor. The proposed sensor in [17] exploits this fringing electric field, which depends on the dimensions of the pores and density of pores (measured per unit area). Choosing optimal pore structure is the key to improve the capacitance of the sensor. Experiments conducted by $\mathrm{Li}$ et al. showed an increase of $60 \%$ in capacitance when $80 \mu \mathrm{m}$ pore size was used. To further increase the capacitance of the sensor, nitrogen plasma is introduced on the surface of the sensor. The wettability of the sensor will increase the immersive area of the sensor, leading to improved fringing fields. Improvement upto 59\% in capacitance was observed when nitrogen plasma was applied over the surface of the sensor.

Chetpattananondh et al. in [18] have developed interdigitated sensor consisting of two conductors with interlocked electrode fingers. The sensor has been fabricated on a FR4 substrate PCB with liquid photoimageable solder mask acting as the water proof coating. When the electrode fingers are exposed to the liquid, capacitance between the electrodes vary. This change in capacitance is measured in the form of discharge curve time of the RC circuit present in the proposed system. The resolution of the system, supported by experimental data, is found out to be at $0.2 \mathrm{~cm}$. The dependence of the system on the type of liquid is eliminated by employing a reference electrode which compensates for the dielectric constant variation and temperature drifts. Further flood-like environment was simulated and the sensor response was evaluated. The problem of hysteresis i.e., tendency to retain the past state, still exists, and is an area of research. The sensor is a contact sensor, and hence over long-term usage, is prone to get corroded.

Kulha et al. [19], Islam et al. in [20] and Ali et al. [21] too have focussed on developing interdigitated capacitive sensors. Kulha et al. in [19] have fabricated four different versions of IDC sensors to comparatively analyze the designs. The system is simple, having STM32F030 microcontroller to process the sensed data. Three external resistors are used apart from the fabricated sensors. The entire system is present on a single screen, which makes the design very cost effective, simple and sleek. The system is capable of temperature measurement too with a resolution of $0.4^{0} \mathrm{C}$. Ali et al. in [21] have proposed a sensor that can work with any type of water. Once the interdigitated sensors convert the change in liquid 
level to a capacitance value, an extensive signal processing system has been used to accurately produce digital readings. The system consists of modified De'Sauty bridge, precision peak rectifier and signal conditioning blocks. The resolution of the system scales down to $12.8 \mathrm{mV}$ for a measuring range of $15 \mathrm{~cm}$. The paper also states that neural networks can be used to process the signal better.

Islam et al. claim that the sensitivity of the liquid level sensing system proposed in [19] is less. Islam et al. have proposed 1-1-1, 1-3-1 and 1-5-1 IDT structures in their work [20]. The sensor system was developed using ANSYS Maxwell 15.0. Capacitance to digital converter (FDC1004) was used to convert the capacitance value to digital readings. Experimental analysis shows that sensitivity is higher when compared to other researchers and the figure stands at $40 \mathrm{fF} / \mathrm{mm}$. Since the sensor system is stuck to the exterior walls of the container, the type of measurement is non-destructive. However, the experiment was carried out for a measurement range of 0-4cm only.

\section{Conclusion}

Various capacitive sensing methods have been investigated in this paper. Two prominent techniques have been observed in capacitive sensing used for liquid level measurements, namely cylindrical sensor geometry and coplanar sensor geometry. Cylindrical sensor geometry has been used in different ways. Some researchers have developed cylindrical sensors consisting of two metallic electrodes. These sensors are then exposed to the liquid so that they can interact with the liquid. The sensors usually involve measuring absolute capacitance or relative capacitance i.e., change in capacitance of the sensor due to the influence of liquid. These define the parameters of measurement namely the self-capacitance and the mutual capacitance respectively. Cylindrical sensor geometry is also implemented using an insulated metallic probe/plate as one electrode, with the insulation acting as the dielectric material. The liquid itself acts as the other electrode and completes the capacitor structure.

Coplanar sensor geometry has been extensively explored in the recent years by the researchers. Comb drive structure and the interdigitated structure are the prominent types of sensor developed under coplanar sensor geometry. This involves interlocked fingers of two electrodes, which are transmitter and receiver electrodes. The presence of water will increase the capacitance between adjacent TX and RX fingers, thus indicating the presence of liquid. Coplanar structures have also been investigated based on the concept of fringing field. The following tables provide the comparison of the various capacitive sensing techniques developed by the researchers. Table 1 gives the comparison of the cylindrical capacitive type sensors. All the researchers cited in Table 1 have used tap water as their test liquid. The coplanar capacitive type sensors have been analyzed and tabulated in Table 2.

\section{Table 1. Comparison of Cylindrical Capacitive Sensors using Tap Water as Test Liquid}

\begin{tabular}{|c|c|c|c|c|}
\hline Ref & Sensor Type & Error \% & Resolution & Sensitivity \\
\hline [9] & Contactless & $\begin{array}{c}\text { Metallic tank: } \\
\text { Glass: } 1.5 \% \\
\text { HDPE: } 4 \% \\
\text { Non-metallic } \\
\text { tank: } \\
\text { Glass: } 0.8 \% \\
\text { HDPE: } 0.6 \%\end{array}$ & $1 \mathrm{~mm}$ & $\begin{array}{c}\text { Glass: } \\
0.95 \mathrm{pF} / \mathrm{mm} \\
\text { HDPE: } \\
0.65 \mathrm{pF} / \mathrm{mm}\end{array}$ \\
\hline [10] & Contactless & $0.275 \%$ & - & $0.4 \mathrm{mV} / \mathrm{cm}$ \\
\hline
\end{tabular}




\begin{tabular}{|c|c|c|c|c|}
\hline [11] & Contact & $0.675 \%$ & $1 \mathrm{~mm}$ & $1.06 \mathrm{pF} / \mathrm{mm}$ \\
\hline$[12]$ & Contact & $0.5 \%$ & - & - \\
\hline
\end{tabular}

Table 2. Comparison of Coplanar Capacitive Sensors

\begin{tabular}{|c|c|c|c|c|}
\hline Ref & Sensor type & Liquid & Resolution & Sensitivity \\
\hline [6] & Contactless & $\begin{array}{l}\text { Red fuming } \\
\text { nitric acid }\end{array}$ & $0.1 \mathrm{~mm}$ & - \\
\hline [16] & Contactless & $\begin{array}{l}\text { Distilled water, } \\
\text { transformer oil }\end{array}$ & - & $0.52 \mathrm{pF} / \mathrm{mm}$ \\
\hline [17] & Contact & $\begin{array}{l}\text { Deionized } \\
\text { water }\end{array}$ & - & $\begin{array}{c}\text { Without N2 } \\
\text { plasma: } \\
\text { 69pF/mm } \\
\text { With N2 plasma: } \\
\text { 97pF/mm }\end{array}$ \\
\hline [18] & Contact & $\begin{array}{l}\text { Pure water, } \\
\text { drinking water, } \\
\text { tap water, } \\
\text { canal water, } \\
\text { seawater, soft } \\
\text { drink }\end{array}$ & $0.2 \mathrm{~mm}$ & $32.28 \mu \mathrm{s} / \mathrm{cm}$ \\
\hline [19] & Contact & Water, oil & $0.1 \mathrm{~mm}$ & $\begin{array}{c}\text { Water: } 0.7 \mathrm{pF} / \mathrm{mm} \\
\text { Oil: } 0.08 \mathrm{pF} / \mathrm{mm}\end{array}$ \\
\hline [20] & Contactless & $\begin{array}{c}\text { Reverse } \\
\text { osmosis water }\end{array}$ & $0.1 \mathrm{~mm}$ & $39.8 \mathrm{fF} / \mathrm{mm}$ \\
\hline [21] & Contact & $\begin{array}{l}\text { Mineral water, } \\
\text { tap water, } \\
\text { distilled water, } \\
\text { river water }\end{array}$ & $12.8 \mathrm{mV}$ & $\begin{array}{c}\text { Mineral water: } \\
\text { 59.69nF/cm } \\
\text { Tap water: } \\
\text { 72.46nF/cm } \\
\text { Distilled water: } \\
\text { 8.13nF/cm } \\
\text { River water: } \\
\text { 197.26nF/cm }\end{array}$ \\
\hline
\end{tabular}

There has not been much research in the staggered coplanar geometry. Staggered geometry involves identical sensors stacked up one above the other. Rectangular shaped sensors were explored by some researchers. Staggered sensors were used at one side of the container and a continuous electrode was employed opposite to the staggered sensor [6]. Electric field lines travel from TX to RX through the liquid. A simpler low power system may be developed where TX and RX are present on the same side of the container. The power supplied may be such that the field lines have sufficient energy to penetrate through the container, and pass through the liquid space. The presence or absence of the liquid dictates the strength of the electric field lines, and thus the capacitance readings. Staggered geometry based capacitive sensors tuned towards liquid level sensing application offer an active area of research.

\section{References}

[1] M. Meribout, M. Habli, A. Al-Naamany, and K. Al-Busaidi, "A new ultrasonic-based device for accuratemeasurement of oil, emulsion, and water levels in oiltanks, " in Proceedings of the 21st IEEE Instrumentationand Measurement Technology Conference (IEEE Cat.No.04CH37510), vol. 3, IEEE, (2004), pp.1942-1947. 
[2] T. Nakagawa, A. Hyodo, K. Osada, H. Kurata, andS. Oho, "Contactless liquid-level measurement throughopaque container using millimeter-wave sensor,” in2011 IEEE SENSORS Proceedings, IEEE, Oct. (2011),pp. 1421-1424.

[3] S. Sonasang, S. Srisawat, R. Phromloungsri, W. Rattanangam,and N. Angkawisittpan, "Liquid level measurementusing sensors with microstrip parallel coupledlines," in 2019 IEEE 2nd International Conference onPower and Energy Applications (ICPEA), IEEE, Apr. (2019).

[4] T. L. Greenwood, "Electronic techniques in liquid levelmeasurement," IRE Transactions on Instrumentation,vol. I-7, no. 1, pp. 65-68, Mar. (1958).

[5] L. Wilner, W. Morrison, and A. Brown, "An instrumentfor measuring liquid level and slosh in the tanks ofa liquid-propellant rocket," Proceedings of the IRE,vol. 48, no. 4, pp. 786-788, Apr. (1960).

[6] F. Toth, G. Meijer, and M. vanderLee, "A new capacitiveprecision liquid-level sensor," in Proceedings of20th Biennial Conference on Precision ElectromagneticMeasurements, (1996), pp. 356-357.

[7] S. Stuchly, M. Hamid, and A. Andres, "Microwavesurface level monitor," IEEE Transactions on IndustrialElectronics and Control Instrumentation, vol. IECI-18,no. 3, pp. 85-92, Aug. (1971).

[8] P. Longrigg, “A laser precision liquid level sensor," IEEE Transactions on Industrial Electronics and ControlInstrumentation, vol. IECI-21, no. 2, pp. 88-96,May (1974).

[9] S. Bera, J. Ray, and S. Chattopadhyay, "A low-costnoncontact capacitance-type level transducer for a conductingliquid," IEEE Transactions on Instrumentationand Measurement, vol. 55, no. 3, pp. 778-786, Jun.(2006).

[10] S. Chakraborty, S. K. Bera, N. Mandal, and S. C. Bera, "Study on further modification of non-contact capacitancetype-level transducer for a conducting liquid,”IEEE Sensors Journal, vol. 15, no. 11, pp. 66786688,Nov. (2015).

[11] B. Jin, Z. Zhang, and H. Zhang, "Structure design andperformance analysis of a coaxial cylindrical capacitivesensor for liquid-level measurement," Sensors and ActuatorsA: Physical, vol. 223, pp. 84-90, Mar. (2015).

[12] S. C. Bera, H. Mandal, S. Saha, and A. Dutta, "Studyof a modified capacitance-type level transducer for anytype of liquid," IEEE Transactions on Instrumentationand Measurement, vol. 63, no. 3, pp. 641-649, Mar.(2014).

[13] S. Bardiya and B. Vasuki, "Performance analysis ofcapacitance-type liquid level measurement at inclinedpositions," in 2017 International Conference on IntelligentComputing, Instrumentation and Control Technologies(ICICICT), IEEE, Jul. (2017), pp. 1736-1739.

[14] L. Guirong and C. Shuyue, "A capacitive liquid levelsensor with four electrodes," in 2010 3rd InternationalConference on Computer Science and Information Technology,vol. 4, IEEE, Jul. (2010), pp. 628-632.

[15] H. Canbolat, “A novel level measurement technique using three capacitive sensors for liquids," IEEE Transactionson Instrumentation and Measurement, vol. 58, no. 10, pp. 3762-3768, Oct. (2009).

[16] A. Pietrikova, S. Zuk, and I. Vehec, "Coplanar capacitiveliquid level sensor," in 2019 42nd InternationalSpring Seminar on Electronics Technology (ISSE),IEEE, May (2019).

[17] Y.-T. Li, C.-M. Chao, and K. Wang, "A capacitancelevel sensor design and sensor signal enhancement," in2011 6th IEEE International Conference on Nano/MicroEngineered and Molecular Systems, IEEE, Feb. (2011).

[18] K. Chetpattananondh, T. Tapoanoi, P. Phukpattaranont,and N. Jindapetch, "A self-calibration water level measurementusing an interdigital capacitive sensor," Sensorsand Actuators A: Physical, vol. 209, pp. 175182,Mar. (2014).

[19] P. Kulha, W. Hilber, A. Laposa, and B. Jakoby, "Screenprinted and laminated electrodes for low-cost capacitivelevel measurement systems," Journal of Electrical Engineering,vol. 69, no. 2, pp. 177-182, Mar. (2018).

[20] T. Islam and O. P. Maurya, "Design and fabrication ofnon-contact fringing field capacitive sensor for liquidlevel measurement," in 2019 IEEE 16th India CouncilInternational Conference (INDICON), IEEE, Dec.(2019), pp. 1-4.

[21] S. F. Ali and N. Mandal, "Design and development of anelectronic level transmitter using inter digital capacitor, ”IEEE Sensors Journal, vol. 19, no. 13, pp. 5179-5185,Jul. (2019). 